B. S. Amurthur and S. A. Prahl, "Acoustic cavitation events during microsecond irradiation of aqueous solutions," Diagnostic and Therapeutic Cardiovascular Interventions VII, R. R. Anderson et al. Ed., Proc. SPIE 2970, 4-9, (1997).

\title{
Acoustic Cavitation Events During Microsecond Irradiation of Aqueous Solutions
}

\author{
B. S. Amurthur, J. A. Viator, S. A. Prahl \\ Oregon Medical Laser Center \\ St. Vincent Hospital, 9205 SW Barnes Rd, Portland, OR 97225
}

\begin{abstract}
The acoustic boundary conditions at the site of laser irradiation affects the onset of cavitation when stress-confined laser pulses are used. This study reports the thresholds for cavitation when the laser pulse length exceeds the stress confinement time by a factor of eight. The cavitation threshold was defined as the loss of an acoustic signal from the collapse of the cavitation bubble in room temperature dye solutions. The threshold temperature increase for cavitation at a free surface was $13 \pm 3^{\circ} \mathrm{C}$, at the tip of a $200 \mu \mathrm{m}$ fiber in an aqueous solution was $17 \pm 2^{\circ} \mathrm{C}$, and at the interface of two acoustically matched boundary materials was $80 \pm 20^{\circ} \mathrm{C}$.
\end{abstract}

Keywords: bubble, ablation, pulse, photospallation, acoustic diffraction

\section{INTRODUCTION}

Ablation studies may be divided into three categories according to the duration of the laser pulse $t_{p}$,

1. Long laser pulses are those that exceed the thermal diffusion time $t_{t h}$ of the tissue,

$$
t_{p}>t_{t h}=\frac{\delta^{2}}{4 \kappa}
$$

where $\delta$ is a characteristic length (penetration depth or spot diameter) and $\kappa$ is the thermal diffusivity of the material. In this case, some or most of the laser energy may diffuse away from the optical absorption site during the laser pulse. The peak temperatures reached are necessarily less than those in the intermediate and short pulse experiments below.

2. Intermediate laser pulses are those that are shorter than the thermal diffusion time and longer than the stress confinement time $t_{c}$ of the material

$$
t_{t h}>t_{p}>t_{c}=\frac{\delta}{c}
$$

where $c$ is the speed of sound in the material. Thermal confinement $t_{p}<t_{t h}$ ensures maximum temperatures, but allows acoustic relaxation during the laser pulse and therefore lower maximum acoustic stresses than for short pulse experiments.

3. Short laser pulses are those that are shorter than the acoustic relaxation time

$$
t_{p}<t_{c} \quad \text { or } \quad \tau_{p}=\frac{t_{p}}{t_{c}}<1
$$

Short laser pulses yield the highest temperatures and acoustic stresses (per unit delivered energy).

Direct correspondence to SAP: prahl@ece.ogi.edu; (503) 216-2197; http://omlc.ogi.edu 

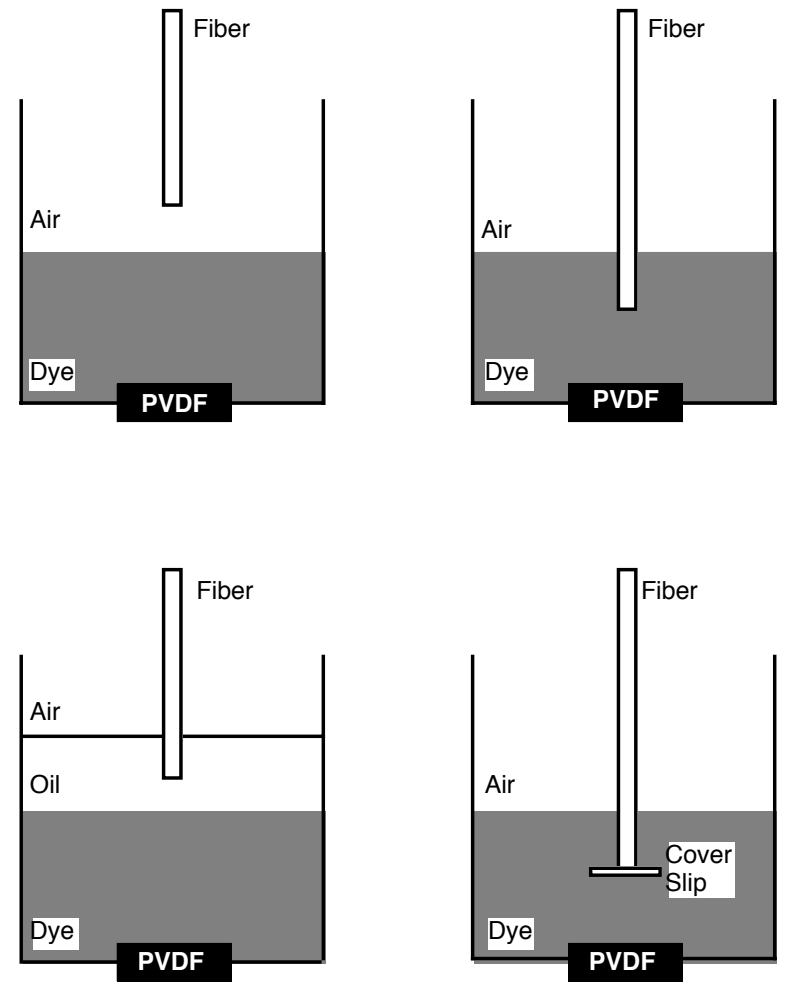

Figure 1. The four experiments used to measure the acoustic threshold for the onset of cavitation.

These three pulse categories are associated with three different types of ablation.

- Vaporization that is achieved by delivering sufficient energy to change the material from a solid to a gas. This is the least efficient mechanism for ablation (in terms of ablated mass per unit energy delivered) and is often accompanied by significant collateral thermal damage. Vaporization is associated with long laser pulses $\left(\tau_{p} \gg 1\right)$.

- Ablation might be caused by mechanical disruption due to cavitation bubble collapse following water vaporization at $100^{\circ} \mathrm{C}$. When a vapor bubble collapses the forces may exceed the strength of the material and remove tissue without vaporizing it. Such material does not need to undergo a phase transition and therefore this process is more efficient than vaporization of all the ablated material. Mechanical disruption is associated with intermediate laser pulses $\left(\tau_{p}>1\right)$.

- Spallation occurs when cavitation bubbles are formed as a consequence of tensile stress waves. ${ }^{1}$ The stress wave breaks mechanical bonds in the material and reduces the temperatures needed for bubble formation. Short pulse ablation studies ${ }^{2-4}(\tau=0.02-0.06)$ have demonstrated bubble formation and acoustic transient detection at temperature increases of $5-10^{\circ} \mathrm{C}$. Photospallation can be efficient and is associated with short laser pulses $\left(\tau_{p}<1\right)$.

We investigated threshold ablation dynamics of intermediate laser pulses during relatively low energy microsecond irradiation. Preliminary experiments with a fiber in a dye solution showed that acoustic transients were detected at fluences as low as $19 \mathrm{~mJ} / \mathrm{mm}^{2}$ and at an absorption coefficient of $5 \mathrm{~cm}^{-1}$ corresponding to a temperature rise of $22^{\circ} \mathrm{C}$. These results led to the question: Are cavitation effects caused by the optical fiber tip? To answer this question experiments were conducted with four different acoustic boundary conditions at the ablation site. These are shown in Figure 1 and are described in the next section. 

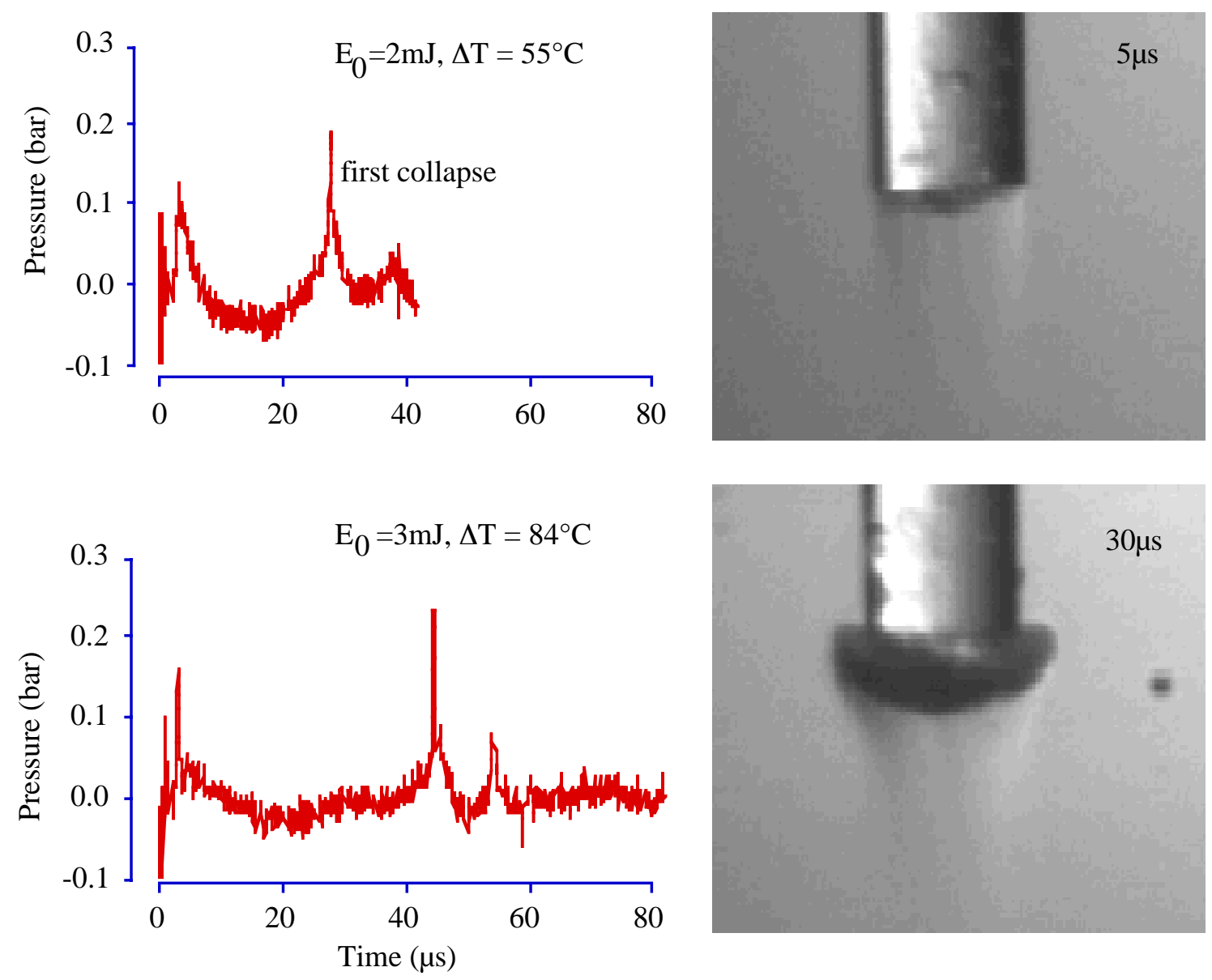

Figure 2. Laser irradiation of aqueous $37 \mathrm{~cm}^{-1}$ solution at $2 \mathrm{~mJ}$ (top) and $3 \mathrm{~mJ}$ (bottom) using a $200 \mu \mathrm{m}$ fiber. The acoustic transients at left show the expansion and collapse of the bubble and a flash photograph at right shows the shape of the bubble. The pressures are significantly less than those in Table 2 because the acoustic transducer was located at a distance from the cavitation site.

\section{MATERIALS AND METHODS}

A flashlamp-pumped pulsed dye laser (Palomar Medical Technologies) was used to irradiate $37 \mathrm{~cm}^{-1}$ aqueous solutions of direct red dye (Sigma). The laser was operated at a wavelength of 522 nm having a pulse duration of $1.4 \mu \mathrm{s}$ (FWHM). The laser light was delivered through a $200 \mu \mathrm{m}$ step index fused silica optical fiber. Neutral density filters were used to obtain pulse energies ranging from $0.4-10 \mathrm{~mJ}$ out of the fiber. The pulse energy was measured with a Joulemeter (JD500, Molectron) before and after each irradiation and varied less than $5 \%$.

Acoustic transients were measured with a shielded PVDF shock transducer (Ktech, 117) having a rise time of $300 \mathrm{~ns}$. The free surface (air-dye interface) was $2 \mathrm{~cm}$ from the transducer. The dye solution was placed in a plastic container $(5 \times 5 \mathrm{~cm})$ with the PVDF transducer fitted into the bottom. The detector sensitivity was $24 \mathrm{mV} / \mathrm{bar}$. The acoustic signals were recorded with a oscilloscope (Tektronix, DSA 602A). All the experiments were carried out at a room temperature. The optical fiber tip was placed $1 \mathrm{~mm}$ away from the transducer surface.

The dye solution also permitted visualization of the bubble formation. A square hole was made on one of the walls of the plastic container and a microscope cover slip was glued on to the window for better imaging. A series of photographs were taken with a triggerable CCD camera (CV 251, Protec). The camera was triggered at variable delay times with respect to the laser pulse. A strobe (MVS 2601, EG\&G Electro-Optics) with a $5 \mu$ s duration was 


\begin{tabular}{|c|c|c|c|c|c|}
\hline $\begin{array}{l}\text { Acoustic } \\
\text { Interface }\end{array}$ & $\begin{array}{c}\text { Ambient } \\
\text { Temperature } \\
(\mu \mathrm{m})\end{array}$ & $\begin{array}{c}\text { Spot } \\
\text { Diameter } \\
(\mathrm{mJ})\end{array}$ & $\begin{array}{c}\text { Threshold } \\
\text { Energy } \\
\left({ }^{\circ} \mathrm{C}\right)\end{array}$ & $\begin{array}{c}\text { Temperature } \\
\text { Increase } \\
\left({ }^{\circ} \mathrm{C}\right)\end{array}$ & $\begin{array}{c}\text { Threshold } \\
\text { Temperature } \\
\left({ }^{\circ} \mathrm{C}\right)\end{array}$ \\
\hline air-dye & 22 & $440 \pm 20$ & $2.5 \pm 0.2$ & $13 \pm 3$ & $35 \pm 3$ \\
\hline oil-dye & 26 & $420 \pm 20$ & $2.5 \pm 1.0$ & $61 \pm 20$ & $81 \pm 20$ \\
\hline fiber-dye & 21 & $200 \pm 10$ & $0.61 \pm 0.03$ & $17 \pm 2$ & $38 \pm 2$ \\
\hline slide-dye & 21 & $260 \pm 20$ & $2.2 \pm 0.1$ & $37 \pm 2$ & $58 \pm 2$ \\
\hline
\end{tabular}

Table 1. Threshold energies and spot sizes for cavitation with various boundary conditions. Threshold temperatures are calculated using the threshold energy, spot size and ambient temperatures.

used to illumination of the target at delay times ranging from 10-5000 $\mu \mathrm{s}$. The strobe times were adjusted using a delay generator (DG535, Stanford Research Systems) that was triggered by a photodiode affixed to the optical fiber.

The threshold for cavitation was determined by following the acoustic transient associated with the first collapse of the cavitation bubble. As the laser energy was reduced, the lifetime of the cavitation bubble was reduced as well. Eventually, the acoustic signal from the collapse dropped below the detection limit for our system. This was (arbitrarily) defined as the threshold for cavitation. The bubble lifetime at threshold was about $20 \mu \mathrm{s}$ and the magnitude was about 30 bar. The initial acoustic expansion was not used because it was often accompanied by electrical noise from the laser.

The acoustic impedance of the dye was taken as that of water $\left(Z_{\mathrm{dye}}=1.5 \mathrm{Ns} / \mathrm{cm}^{3}\right.$. The acoustic impedance of the other materials is listed in Table 2 . The spot size was measured at the site of laser absorption by ablating thermally sensitive black paper at $1 \mathrm{~mm}$ and near contact $(\sim 50 \mu \mathrm{m})$.

Laser irradiation was performed in four different geometries to assess the affect of the acoustic boundary conditions on the threshold for cavitation bubble formation (Figure 2). The relative acoustic impedance mismatch at the laser absorption site was set to less than, approximately equal to, and greater than one. This was done by irradiating a free surface of the dye solution in air (the air-dye case), irradiating onto an oil-dye interface (the oil-dye case), and immersing the fiber in the dye to irradiate at the fiber face (the fiber-dye case). A final experiment was done with a microscope cover slip placed on the surface of the fiber so that the $200 \mu \mathrm{m}$ fiber tip could not influence the boundary (this was the slide-dye case).

\section{RESULTS}

The two characteristic distances in our experimental setup are the optical penetration distance $(270 \mu \mathrm{m})$ and the fiber diameter or spotsize $(>200 \mu \mathrm{m})$. The characteristic distance is therefore $270 \mu \mathrm{m}$ and therefore $\tau \approx 8$ and the stress attenuation factor was about 0.13 .

The expansion and first collapse pressure transients were reproducible. Laser flash photographs shown in Figure 2 confirmed creation of small bubbles at a temperature increase of $55^{\circ} \mathrm{C}$. The bubble formation is limited to the front surface of the fiber. The bubbles for irradiances associated with a temperature increase of $75^{\circ} \mathrm{C}$ were larger and longer lived.

The temperature increase induced at the surface by a radiant exposure $E_{0}$ in a sample having absorption coefficient of $\mu_{a}$ for a thermally confined laser pulse is

$$
\Delta T=\frac{\mu_{a} E_{0}}{\rho c_{v}}
$$

where $c_{v}$ is the heat capacity at constant volume. By adding the temperature increase $\Delta T$ to the ambient temperature, the threshold temperature is obtained. The threshold temperatures in Table 1 indicate that both the air-dye, fiberdye, and slide-dye geometries have thresholds significantly below the $100^{\circ} \mathrm{C}$ boiling point of water. Evidently, another physical property is affecting the onset of cavitation in these cases.

The results for various acoustic boundary conditions are shown in Table 1. During the experiments done with the fiber submerged in the oil floating above the dye, acoustic transients were detected only at fluences that corresponded 


\begin{tabular}{|c|c|c|c|c|c|c|}
\hline $\begin{array}{l}\text { Acoustic } \\
\text { Interface }\end{array}$ & $\begin{array}{c}Z \\
\left(\mathrm{Ns} / \mathrm{cm}^{3}\right)\end{array}$ & $\frac{Z-Z_{\text {dye }}}{Z+Z_{\text {dye }}}$ & $\frac{1-\exp \left(-\tau_{p}\right)}{\tau_{p}}$ & $\Gamma$ & $\begin{array}{l}\mu_{a} E_{0} \\
\text { (bar) }\end{array}$ & $\begin{array}{c}\sigma_{\text {refl }} \\
\text { (bar) }\end{array}$ \\
\hline air-dye & 0.33 & -0.64 & 0.13 & 0.17 & $550 \pm 100$ & $-4 \pm 1$ \\
\hline oil-dye & 1.4 & 0.03 & 0.13 & 0.40 & $2600 \pm 1000$ & $4 \pm 1$ \\
\hline fiber-dye & 13.1 & 0.82 & 0.13 & 0.18 & $700 \pm 100$ & $7 \pm 1$ \\
\hline slide-dye & 11.4 & 0.77 & 0.13 & 0.35 & $1550 \pm 100$ & $27 \pm 3$ \\
\hline air-dye & 0.33 & -0.64 & 1.00 & 0.13 & 210 & -9 \\
\hline
\end{tabular}

Table 2. Magnitude of the reflected wave in different experimental conditions. The threshold $\mu_{a} E_{0}$ in the last line was for $14 \mathrm{~ns}$ ablation of an aqueous target at a free surface. ${ }^{2}$

to a temperature rise of $60 \pm 20^{\circ} \mathrm{C}$. However, during irradiation with the fiber tip placed above the dye solution transients signals were detected at temperature rises as low as $13 \pm 2^{\circ} \mathrm{C}$. Furthermore when a bare $200 \mu \mathrm{m}$ fiber tip was submerged in the dye solution, the threshold rise was $17 \pm 2^{\circ} \mathrm{C}$. When the dye solution was irradiated with the fiber attached to the glass no transients were observed until a temperature increase of $37 \pm 2^{\circ} \mathrm{C}$ was reached.

\section{DISCUSSION}

An acoustic impedance mismatch affects how the pressure waves are reflected from the boundary. As the waves travel from the dye solution to air, some of the positive pressure wave will be reflected back as a tensile wave. For a matched medium the boundary should have little or no effect upon the pressure wave and it will travel across the boundary undisturbed. Finally, when travelling from the dye solution into glass, some of the pressure wave will be reflected back from the boundary as a positive compression wave. The magnitude of the reflected stress wave $\sigma_{\text {refl }}$ is 4,5

$$
\sigma_{\text {refl }}=\frac{Z-Z_{\text {dye }}}{Z+Z_{\text {dye }}} \cdot \frac{\Gamma \mu_{a} E_{0}}{2} \cdot \frac{1-\exp \left(-\tau_{p}\right)}{\tau_{p}},
$$

where $Z$ and $Z_{\text {dye }}$ are the acoustic impedances of the material over the dye solution and of the dye (i.e., water) respectively. The Grüneisen parameter $\Gamma$ for water is a function of the temperature $T$

$$
\Gamma=0.0325+0.003875 T \text {. }
$$

The factor of two arises because the stress propagates in opposite directions along the laser beam axis each with half the initial magnitude.

The relevant stress wave values for the four acoustic boundaries are tabulated in Table 2. A negative value for $\sigma_{\text {refl }}$ indicates that the pressure changes signs (from a compression wave to a tensile wave). Now if a reflected tensile stress wave alters the threshold for cavitation, then one would expect the air-dye interface to have a threshold less than $100^{\circ} \mathrm{C}$ and all others to have thresholds near $100^{\circ} \mathrm{C}$. Furthermore, the magnitude of the reflected stress wave should be comparable to the tensile wave arising from short pulse threshold measurements. Oraevsky et al. showed that a $14 \mathrm{~ns}$ YAG laser pulse induced cavitation effects (in a $20^{\circ} \mathrm{C}$ solution with an air-water interface) at a $5^{\circ} \mathrm{C}$ temperature rise. ${ }^{2}$ As can be seen in Table 2, this created a tensile wave that was roughly twice that calculated for the intermediate duration pulses $\left(\tau_{p}=8\right)$. Evidently the tensile waves produced in the air-dye geometry with the long pulse laser are sufficient to lower the cavitation threshold significantly below $100^{\circ} \mathrm{C}$.

The low threshold in the fiber-dye geometry is apparently enhanced by the presence of the edges of the small fiber. When a microscope cover slip is placed at the tip of the fiber, the calculated threshold temperature rises significantly (Table 1). This suggests that diffraction effects from the edges of the fiber may produce tensile waves. ${ }^{6}$ Because the threshold temperature in the presence of a cover slip is also less that $100^{\circ} \mathrm{C}$, diffraction effects are also present (to a lesser extent) when the acoustic boundary is much larger than the spot size. 


\section{ACKNOWLEDGEMENTS}

We wish to thank Steve Jacques for his valuable suggestions. We also wish to thank Alan Shearin, Ujwal

Sathyam, and HanQun Shangguan for their support and input. This work was supported by the Murdock and Whitaker foundations.

\section{REFERENCES}

1. R. S. Dingus, D. R. Curran, A. A. Oraevsky, and S. L. Jacques, "Microscopic spallation process and its potential role in laser-tissue ablation," in SPIE Proceedings of Laser Tissue Interaction V, S. L. Jacques, ed., vol. 2134, 1994.

2. A. A. Oraevsky, S. L. Jacques, and F. K. Tittel, "Mechanism of laser ablation for aqueous media irradiated under stress confined conditions," J. Appl. Phys. 78, pp. 1281-1289, 1995.

3. V. Venugopalan, A. D. Zweig, T. F. Deutsch, N. S. Nishioka, and B. B. Mikić, "Physical mechanisms controlling the generation of laser-induced stresses," in SPIE Proceedings of Laser-Tissue Interaction V, S. L. Jacques, ed., vol. 1882, pp. 102-111, 1993.

4. G. Paltauf and H. Schmidt-Kloiber, "Model study to investigate the contribution of spallation to pulsed laser ablation of tissue," Lasers Surg. Med. 16, pp. 277-287, 1995.

5. R. S. Dingus and R. J. Scammon, "Grüneisen-stress induced ablation of biological tissue," in SPIE Proceedings of Laser-Tissue Interaction II, S. L. Jacques, ed., vol. 1427, pp. 45-54, 1991.

6. G. Paltaulf, M. Frenz, and H. Schmidt-Kloiber, "Influence of acoustic diffraction on laser-induced stress wave effects in absorbing media," in SPIE Proceedings of Laser-Tissue Interaction VII, S. L. Jacques, ed., vol. 2681, pp. 334-342, 1996. 